Frédéric Mallein-Gerin Michel Van der Rest

\section{ADRESSES}

F. Mallein-Gerin : chargé de recherches au Cinrs. UPR 412, Institut de Biolo ie et Chimie des Protéines, 7, passage du Vercors, 69367 Lyon Cedex 07, France. M. Van der Rest professeur à l'Ecole Normale Supérieure de Lyon, directeur du laboratoire de structure et ingénierie des collagènes de l'Institut de Biologie el Chimie des proteines. Institut de Biologie Structurale

J.P. Ebel, 4l, avenue des Martyrs, 38027

Grenoble Cedex 01, France.

\title{
La culture de chondrocytes: outil d'analyse de la différenciation et de l'organisation moléculaire du cartilage
}

La recherche des gènes impliqués dans la régulation de la prolifération et de la maturation des chondrocytes se heurte aux difficultés d'extraction d'ARN à partir de cartilage, et à la perte du phénotype chondrocyte en culture traditionnelle sur plastique. Des méthodes de culture en suspension et des stratégies d'immortalisation ont été développées, qui ont servi à analyser la synthèse et l'assemblage de molécules de structure du cartilage. L'utilisation d'oncogènes a révélé que prolifération et maturation sont, pour les chondrocytes, deux phénomènes mutuellement exclusifs. En outre, l'immortalisation de chondrocytes humains permet de constituer un réservoir cellulaire précieux pour des études pharmacologiques et ouvre la voie à l'immortalisation de chondrocytes de cartilage pathologique. Ces lignées devraient permettre de cloner plus facilement les gènes contrôlant les différents étapes de différenciation du chondrocyte, ou impliqués dans le maintien ou le dérèglement du phénotype cartilagineux.

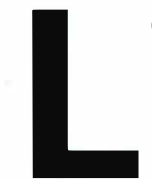

es chondrocytes sont des cellules spécialisées qui dérivent des cellules mésenchymateuses durant le développement embryonnaire pour former les différents cartilages des vertébrés. Chez l'homme, on distingue trois types de cartilage, selon la nature de la matrice extracellulaire. (1) Le cartilage hyalin, le plus abondant, est constitué d'une matrice extracellulaire importante riche en collagènes et protéogly- canes, synthétisée par un nombre relativement restreint de cellules. Dans ce tissu, la force tensile des fibres de collagène s'oppose à la pression osmotique due à la grande densité de charge des protéoglycanes pour former un système biologique amortisseur de chocs au niveau des surfaces articulaires. (2) Le fibrocartilage est un intermédiaire entre un tissu conjonctif et un cartilage hyalin. Il est riche en fibres de collagène qui lui confèrent une très grande résis- 


\section{RÉFÉRENCES}

1. Bruckner P, Van der Rest M. Structure and function of cartilage collagens. Micr Res Tech 1994; 28 : 378-84.

2. Van der Rest $M$. Biologie du collagène et maladies héréditaires de la matrice extracellulaire. médecine/sciences 1987; 3: 411-20.

3. Hall BK Cartilage. New York: Academic Press, 1983: 181-251.

4. Gibson GJ, Kohler WJ, Schaffler MB. Chondrocyte apoptosis in endochondral ossification of chick sterna. Dev Dyn 1995; 203: 468-76.

5. Gentili C, Bianco P, Neri M, Malpeli M, Campanile G, Castagnola P, Cancedda R, DescalziCancedda F. Cell proliferation, extracellular matrix mineralization, and ovotransferrin transient expression during in vitro differentiation of chick hypertrophic chondrocytes into osteoblast-like cells. J Cell Biol 1993; 122: 703-12.

6. Roach II, Erenpreisa J, Aigner T. Osteogenic differentiation of hypertrophic chondrocytes involves asymetric cell divisions and apoptosis. J Cell Biol 1995; 131: 483-94.

7. Maroteaux P. Les maladies osseuses de l'enfant, $3^{e}$ ed. Paris: Flammarion, 1995.

8. Coppin C, Eeckout Y. L'ostéogenèse imparfaite: des mutations aux phénotypes. médecines/sciences $1995 ; 11$ : 853-9.

9. Briggs MD, Hoffman SMG, King LM, Olsen AS, Mohrenweiser H, Leroy JG, Mortier GR, Rimoin DL, Lachman RS, Gaines ES, Cekleniak JA, Knowlton RG, Cohn DH. Pseudoachondroplasia and multiple epiphyseal dysplasia due to mutations in the cartilage oligomeric matrix protein gene. Nature Genet $1995 ; 10: 330-6$.

10. Rousseau F, Bonaventure J, Legeai-Mallet L, Pelet A, Rozet JM, Maroteaux P, Le Merrer $M$, Munnich A. Mutations in the gene encoding fibroblast growth factor receptor-3 in achondroplasia. Nature $1994 ; 371$ : 252-4.

11. Schipani E, Kruse E, Jüppner H. A constitutively active mutant PTH-PTHrP receptor in Jansen-type metaphyseal chondrodysplasia. Science 1995; 268: 98-100.

12. Hästbacka J, de la Chapelle A, Mahtani MM, Clines G, Reeve-Daly MP, Daly M, Hamilton BA, Kusumi K, Trivedi B, Weaver A, Coloma A, Lovett M, Buckler A, Kaitila I, Lander ES. The diastrophic dysplasia gene encodes a novel sulfate transporter: positional cloning by fine-structure linkage disequilibrium mapping. Cell 1994; 78: 1073-87.

13. Franco B, Meroni G, Parenti G, Levilliers J, Bernard L, Gebbia M, Cox L, Maroteaux P, Sheffield L, Rappold GA, Andria G, Petit C, Ballabio A. A cluster of sulfatase genes on Xp22.3: mutations in chondrodysplasia punctata (CDPX) and implications for warfarin embryopathy. Cell 1995; 81 : 15-25.

14. Thomas JT, Lin K, Nandedkar MA, McBride W, Camargo M, Cervenka J, Luy ten FP. A human chondrodysplasia due to a mutation in a TGF- $\beta$ superfamily member. Molecular and developmental biology of cartilage meeting. The New York Academy of Sciences, Bethesda, Maryland, 1995.

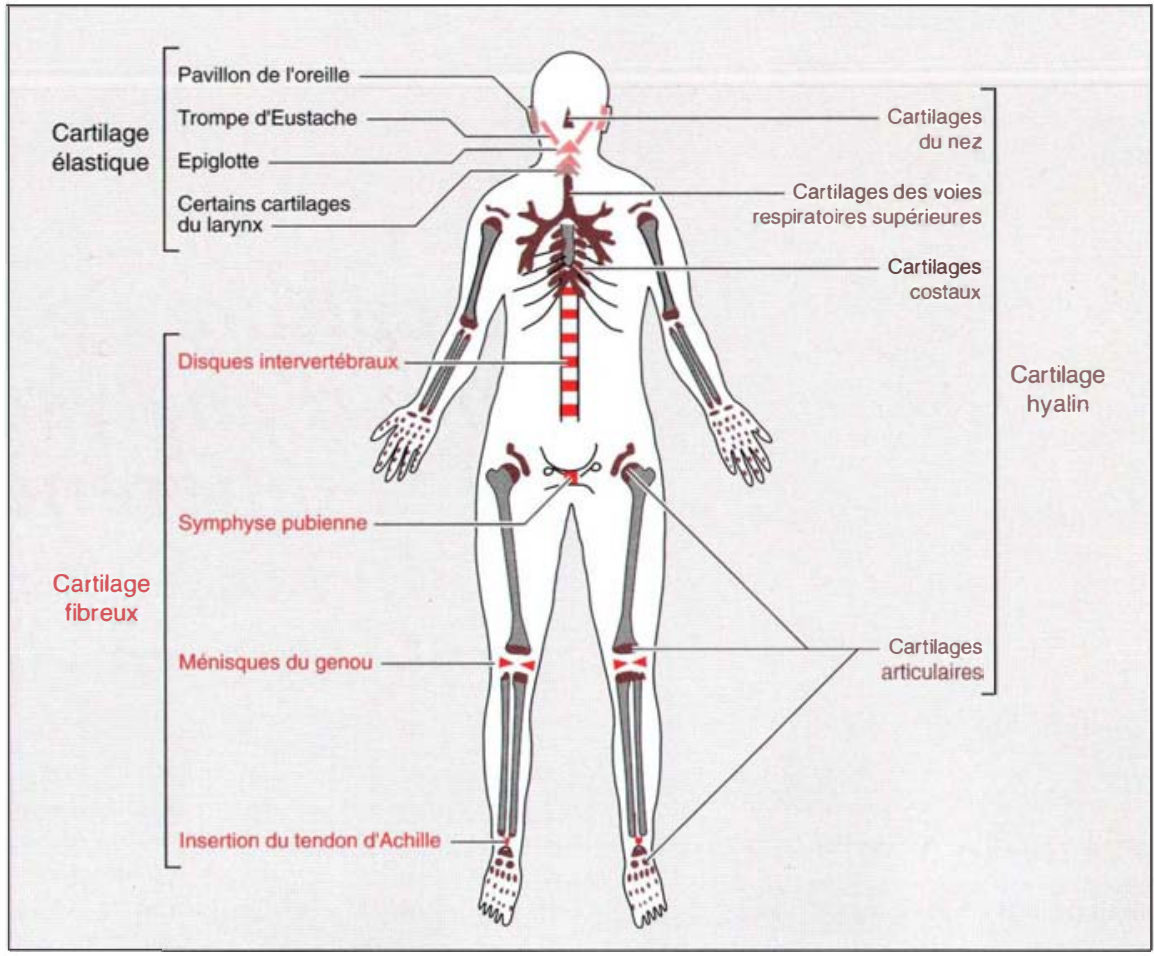

Figure 1. Topographie des cartilages chez l'homme adulte. (D'après [60].)

tance aux tractions et compressions et constitue les zones d'insertion de certains tendons et les disques intervertébraux. (3) Le cartilage élastique est caractérisé par la présence de fibres élastiques et constitue, par exemple, le pavillon de l'oreille (figure 1).

Au cours du développement embryonnaire des vertébrés, les cellules mésenchymateuses préchondrogéniques se condensent pour se différencier en chondrocytes. Cette différenciation se traduit par la production de protéines caractéristiques comme les collagènes II, IX et XI [ 1 , 2] et le protéoglycane agrécane [3] (figure 2). Ce phénotype est maintenu de façon stable dans les cartilages permanents comme le cartilage articulaire. Cependant, la formation de cartilage est une étape nécessaire mais transitoire lors de l'ossification endochondrale. Durant ce processus complexe, les chondrocytes poursuivent leur différenciation en s'hypertrophiant. Cette maturation cellulaire est caractérisée par la synthèse d'un collagène spécifique, le collagène $\mathrm{X}$ [1], alors que la matrice extracellulaire se calcifie progressivement (figure 3). Les chondrocytes hypertrophiques entrent ensuite en apoptose
[4], facilitant l'invasion par les ostéoblastes, et la formation d'une nouvelle matrice osseuse (figure 4). L'apoptose n'est peut-être pas la seule destinée du chondrocyte hypertrophique qui pourrait s'engager dans une voie ultérieure de différenciation: des chondrocytes hypertrophiques peuvent se différencier in vitro en cellules de type ostéoblastique [5]. Une division asymétrique des chondrocytes hypertrophiques a été récemment mise en évidence dans des cultures de fémurs d'embryons de poulet, où une cellule fille meurt par apoptose et l'autre cellule fille se divise en engendrant des cellules ostéoblastiques [6]. La synthèse de protéines caractéristiques de l'os (collagène I, ostéopontine, ostéonectine, sialoprotéine de l'os) par les chondrocytes hypertrophiques a aussi été détectée in vivo par immunohistochimie ou hybridation in situ.

Des mutations dans des gènes codant pour des protéines de structure comme les collagènes $[7,8]$ ou la protéine oligomérique de la matrice du cartilage $\left(\mathrm{m} / \mathrm{s} n^{\circ} 10\right.$, vol. 11 , p. 1499) [9] ont déjà été identifiées dans plusieurs cas d'ostéochondrodysplasies. Mais d'autres mutations affectant des gènes codant pour des 


\section{RÉFÉRENCES}

15. Wagner T, Wirth J, Meyer J, Zabel B, Held M, Zimmer J, Pasantes J, Bricarelli F. D, Keutel J, Hustert E, Wolf U, Tommerup N, Schemp W, Scherer G. Autosomal sex reversal and campomelic dysplasia are caused by mutations in and around the SRYrelated gene SOX9. Cell 1994; 79: 1111-20.

16. Nerlich AG, Wiest I, von der Mark K Immunohistochemical analysis of interstitial collagens in cartilage of different stages of osteoarthrosis. Virchows Arch B Cell Pathol $1993 ; 63: 249-55$.

17. Mallein-Gerin F, Ruggiero F, Garrone R. Proteoglycan core protein and type II collagen gene expressions are not correlated with cell shape changes during low density chondrocyte cultures. Differentiation 1990 ; 43: 204-11

18. Mallein-Gerin F, Garrone R, Van der Rest M. Proteoglycan and collagen synthesis are correlated with actin organization in dedifferentiating chondrocytes. Eur J Cell Biol $1991 ; 56: 36473$.

19. Benjamin M, Archer CW, Ralphs JR. Cytoskeleton of cartilage cells. Microsc Res Tech 1994; 28 : 372-7.

20. Freyria AM, Ronzière MC, Boutillon MM, Herbage D. Effect of retinoic acid on protein synthesis by foetal bovine chondrocytes in high-density culture: down-regulation of the glucose-regulated protein, GRP78 , and type II collagen. Biochem J 1995 305: 391-6.

21. Bates GP, Schor SL, Grant ME. A comparison of the effects of different substrata on chondrocyte morphology and the synthesis of collagen types IX and X. In vitro Cell Dev Biol 1987; 23: 374-80.

22. Watt FM, Dudhia J. Prolonged expression of differentiated phenotype by chondrocytes cultured at low density on a composite substrate of collagen and agarose that restricts cell spreading. Differentiation $1988 ; 38: 140-7$

23. Reginato AM, Iozzo RV, Jimenez SA Formation of nodular structures resembling mature articular cartilage in long-term primary cultures of human fetal epiphysea chondrocytes on a hydrogel substrate. Arthritis Rheum 1994; 37: 1338-49.

24. Horton W, Hassel JR. Independence of cell shape and loss of cartilage matrix production during retinoic acid treatment of cultured chondrocytes. Dev Biol 1986; 115 . 392-7.

25. Bassleer C, Gysen P, Bassleer R, Franchimont $P$. Effects of peptidic glycosaminoglycans complex on human chondrocytes cultivated in three dimensions. Biochem Pharmacol 1988; 37: 1939.

26. Gibson GJ, Schor SL, Grant ME. Effects of matrix macromolecules on chondrocyte gene expression: synthesis of a low molecular weight collagen species by cells cultured within collagen gels. I Cell Biol 1982; 93 . 767-74.

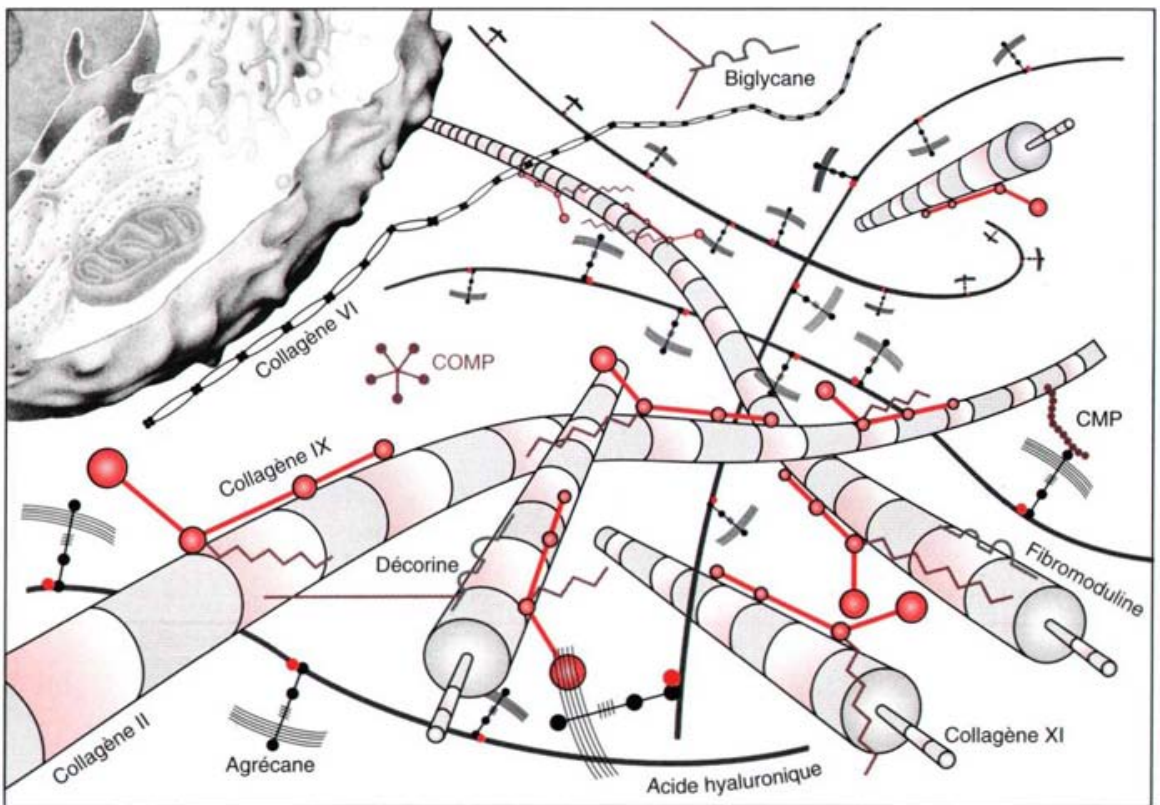

Figure 2. Illustration schématique des constituants du cartilage Inon hypertrophique), et de leurs interactions possibles. Les trois types majeurs de molécules sont les collagènes, les protéoglycanes (agrécanes), et les glycoprotéines non collagéniques. Les fibres sont constituées des collagènes II, IX et XI. Les protéines de liaison stabilisent les agrécanes sur I'acide hyaluronique. Toutes les molécules ne sont pas représentées à l'échelle et certaines interactions proposées ici ont été reprises d'après [61]. COMP: cartilage oligomeric matrix protein, CMP: cartilage matrix protein.

protéines aussi diverses qu'un récepteur de facteur de croissance $(\mathrm{m} / \mathrm{s}$ $n^{\circ} 8-9$, vol. 10, p. 936) [10], un récepteur de peptide relié à l'hormone parathyroïdienne $\left(\mathrm{m} / \mathrm{s} n^{\circ} 6\right.$, vol. 11 , p. 920) [11], un transporteur de sulfate $\left(\mathrm{m} / \mathrm{s} n^{\circ} 10, v o l .11, p .1492\right)$ [12], une sulfatase $\left(\mathrm{m} / \mathrm{s} n^{\circ} 10\right.$, vol. 11 , p. 1494) [13], un facteur de différenciation [14], un facteur de transcription potentiel $\left(\mathrm{m} / \mathrm{s} n^{\circ} 2\right.$, vol. $11, p$. 300) [15], conduisent aussi à des perturbations de la squelettogenèse (Tableau I). D'autres gènes sont vraisemblablement impliqués dans la régulation de la prolifération et des étapes de détermination, différenciation et maturation des chondrocytes. Dans la maladie d'Ollier, la prolifération non contrôlée des chondrocytes est à l'origine d'ostéochondromes ou de chondromes qui peuvent produire des sarcomes [7]. Il est aussi connu qu'au cours d'affections dégénératives du cartilage comme l'arthrose, les lésions mécaniques de la matrice cartilagineuse sont associées à des anomalies morphologiques et fonctionnelles des chondrocytes. Une dédifférenciation cellulaire (expression par le chondro- cyte de collagènes de types I et III, "de type fotal") participerait à l'entretien du processus pathologique [16]. Ainsi, la connaissance des gènes impliqués dans l'apparition et/ou le maintien du phénotype chondrocytaire, mais aussi dans la prolifération et la maturation des chondrocytes permettrait de mieux comprendre les mécanismes cellulaires participant à ces différents processus pathologiques. Durant ces dernières années, le contrôle qu'exerce l'environnement sur le phénotype des chondrocytes a été abondamment étudié et de nombreux facteurs ont été identifiés (facteurs de croissance, hormones, vitamines, matrice extracellulaire). En revanche, la recherche de gènes impliqués dans la régulation de ce phénotype se heurte aux difficultés d'extraction d'ARN à partir de cartilage, et à l'instabilité du chondrocyte en culture classique sur plastique. Nous allons présenter ici différentes techniques de culture permettant de conserver le phénotype du cartilage, ainsi que des stratégies d'immortalisation de chondrocytes qui ont servi à mieux comprendre l'assemblage, 


\begin{tabular}{|c|c|c|}
\hline \multicolumn{3}{|c|}{ Tableau I } \\
\hline \multicolumn{3}{|c|}{$\begin{array}{c}\text { OSTÉOCHONDRODYSPLASIES } \\
\text { (NON ASSOCIÉES À D'AUTRES SYMPTÔMES) PROVOQUÉES PAR DES MUTATIONS CHEZ L'HOMME }\end{array}$} \\
\hline Types de protéine & $\begin{array}{l}\text { Ostéochondrodysplasies } \\
\text { provoquées par mutation }\end{array}$ & Références \\
\hline $\begin{array}{l}\text { Protéine de structure } \\
\alpha 1(1) \text { et } \alpha 2(I) \text { collagène }\end{array}$ & Ostéogenèse imparfaite & [8] \\
\hline$\alpha 1$ (II) collagène & $\begin{array}{l}\text { Syndrome de Stickler } \\
\text { Dysplasie spondylo-épiphysaire congénitale } \\
\text { Achondrogenèse \| de Langer-Saldino } \\
\text { Hypochondrogenèse } \\
\text { Dysplasie spondylo-épimétaphysaire } \\
\text { Dysplasie de Kniest } \\
\text { Spondylo-arthropathies familiales }\end{array}$ & [7] \\
\hline$\alpha 2(I X)$ collagène & Dysplasie épiphysaire multiple & $\mathrm{m} / \mathrm{s} n^{\circ} 10$, vol. 11, p. 1499 \\
\hline$\alpha 2(X I)$ collagène & Syndrome de Stickler & $\mathrm{m} / \mathrm{s} n^{\circ} 10$, vol. 12, p. 1171 \\
\hline$\alpha 1(X)$ collagène & Chondrodysplasie métaphysaire de Schmid & [7] \\
\hline COMP & Pseudo-achondroplasie & $\mathrm{m} / \mathrm{s} n^{\circ} 10$, vol. 11, p. 1499 \\
\hline $\begin{array}{l}\text { Récepteur } \\
\text { FGFR3 }\end{array}$ & $\begin{array}{l}\text { Nanismes thanatophores I et II } \\
\text { Achondroplasie } \\
\text { Hypochondroplasie }\end{array}$ & $\begin{array}{l}\mathrm{m} / \mathrm{s} n^{\circ} 5, \text { vol. } 11, p .730 \\
\mathrm{~m} / \mathrm{s} n^{\circ} 8-9, \text { vol. } 10, p .936 \\
\mathrm{~m} / \mathrm{s} n^{\circ} 4, \text { vol. } 10, \text { p. } 486\end{array}$ \\
\hline Récepteur PTH-PTHrP & Chondrodysplasie métaphysaire de Jansen & $\mathrm{m} / \mathrm{s} n^{\circ} 6$, vol. 11, p. 920 \\
\hline $\begin{array}{l}\text { Transporteur } \\
\text { Transporteur } \\
\text { de sulfate }\end{array}$ & $\begin{array}{l}\text { Dysplasie diastrophique } \\
\text { Achondrogenèse IB } \\
\text { Atélostéogenèse II }\end{array}$ & $\mathrm{m} / \mathrm{s} n^{\circ} 6$, vol. 12, p. 833 \\
\hline $\begin{array}{l}\text { Enzyme } \\
\text { Arylsulfatase } E\end{array}$ & Chondrodysplasie ponctuée & $\mathrm{m} / \mathrm{s} n^{\circ} 10$, vol. 11, p. 1492 \\
\hline $\begin{array}{l}\text { Facteur de différenciation } \\
\text { CDMP-1 }\end{array}$ & $\begin{array}{l}\text { Chondrodysplasie acromésomélique } \\
\text { (Hunter-Thompson) }\end{array}$ & [7] \\
\hline $\begin{array}{l}\text { Facteur de transcription } \\
\text { SOX9 }\end{array}$ & Dysplasie campomélique & $\mathrm{m} / \mathrm{s} n^{\circ} 2$, vol. 11, p. 300 \\
\hline
\end{tabular}

CDMP : cartilage-derived morphogenetic protein ; PTH-PTHrP: parathormone, PTH related protein.

l'organisation de macromolécules de la matrice cartilagineuse, et la relation existant entre prolifération cellulaire et maturation du chondrocyte.

\section{Modulation phénotypique du chondrocyte in vitro}

Techniques de culture

Le choix de la culture cellulaire de chondrocytes comme modèle expéri- lularité du cartilage, qui limite les méthodes d'étude in vivo. Les chondrocytes, une fois isolés de leur matrice, peuvent être cultivés sur un substrat ou en suspension (figure 5). La culture des chondrocytes en monocouche sur plastique a été largement exploitée, car c'est une méthode facile qui permet une prolifération active des chondrocytes. Toutefois, elle induit une «modulation phénotypique» ou «dédifférenciation ", caractérisée par le passage d'une forme cellulaire arrondie à une forme fibroblastique, et par une diminution progressive de la synthèse des collagènes II, IX, XI et de l'agrécane, et l'apparition des collagènes I, III et V. Cependant, cette perte du phénotype n'est pas directement dépendante de la forme cellulaire, mais est en relation étroite avec l'organisation du cytosquelette d'actine $[17,18]$. La matrice extracellulaire synthétisée en monocouche et son interaction au niveau 


\section{RÉFÉRENCES}

27. Benya PD, Schaffer JD. Dedifferentiated chondrocytes reexpress the differentiated chondrocyte phenotype when cultured in agarose gels. Cell 1982; 30 : 215-24.

28. Castagnola P, Moro G, Descalzi-Cancedda $F$, Cancedda $R$. Type X collagen synthesis during in vitro development of chick embryo tibial chondrocytes. J Cell Biol 1986; 102: $2310-7$.

29. Horton WA, Machado MA, Ellard J, Campbell D, Bartley J, Ramirez F, Vitale E, Lee $B$. Characterization of a type II collagen gene (COL2A1) mutation identified in cultured chondrocytes from human hypochondrogenesis. Proc Natl Acad Sci USA 1992; 89: 4583-7.

30. Freisinger $P$, Stanescu V, Jacob B, Cohen-Solal L, Maroteaux P, Bonaventure J. Achondrogenesis type IB (Fraccaro): study of collagen in the tissue and in chondrocytes cultured in agarose. Am J Med Genet $1994 ; 49: 439-46$

31. Häuselmann $\mathrm{HJ}$, Aydelotte MB, Schumacher BL, Kuettner KE, Gitelis SH, Thonar EJ. Synthesis and turnover of proteoglycans by human and bovine adult articular chondrocytes cultured in alginate beads. Matrix 1992; 12: 116-29.

32. Bonaventure J, Khadom N, Cohen-Solal $\mathrm{L}, \mathrm{Ng} \mathrm{KH}$, Bourguignon J, Lasselin C, Freisinger $\mathrm{P}$. Reexpression of cartilage-specific genes by dedifferentiated human articular chondrocytes cultured in alginate beads. Exp Cell Res 1994; 212: 97-104.

33. Lafeber FPJG, Vander Kraan PM, Van Roy AM, Huber-Bruning $O$, Bijlsma JWJ. Articular cartilage explant culture; an appropriate in vitro system to compare osteoarthritic and normal human cartilage. Connect Tissue Res 1993; 29: 287-99.

34. Cancedda R, Cancedda FD, Castagnola $\mathrm{P}$. Chondrocyte differentiation. Int Rev Cyt 1995 ; 159: 265-358.

35. Böhme K Winterhalter KH, Bruckner P. Terminal differentiation of chondrocytes in culture is a spontaneous process and is arrested by transforming growth factor- $\beta 2$ and basic fibroblast growth factor in synergy. Exp Cell Res 1995; 216: 191-8.

36. Sautier J, Forest N. Les protéines de la morphogenèse osseuse: BMP. médecine/ sciences $1996 ; 12: 36470$.

37. Konieczny SF, Emerson CP, Jr. 5-Azacytidine induction of stable mesodermal stem cell lineages from 10T1/2 cells: evidence for regulatory genes controlling determination. Cell 1984;38: 791-800.

38. Wang EA, Israel DI, Kelly S, Luxenberg DP. Bone morphogenetic protein- 2 causes commitment and differentiation in C3H10T1/2 and 3T3 cells. Growth Factors $1993 ; 9$ : $57-71$.

39. Ahrens M, Ankenbauer T, Schroder D, Hollnagel A, Mayer H, Gross G. Expression of human bone morphogenetic proteins-2 or -4 in murine mesenchymal progenitor C.3H10T1/2 cells induces differentiation into distinct mesenchymal cell lineages. DNA Cell Biol 1993; 12: 871-80. des plaques d'adhérence au cytosquelette produit vraisemblablement une cascade d'inductions qui conduisent à l'altération de l'expression génétique du chondrocyte [19].

Sur plastique, on peut retarder cette dédifférenciation en cultivant les chondrocytes à forte densité. Ils forment, avec le temps, des couches cellulaires multiples, enrobées dans une matrice similaire à celle trouvée in vivo [20]. Des substrats servant d'interface entre le plastique et les cellules ont été aussi utilisés pour retarder ou éviter la dédifférenciation, comme un film de collagène [21], un composite de collagène et d'agarose [22] ou un gel de méthacrylate [23]. Ces études ont montré que la matrice extracellulaire environnante exerçait un contrôle sur le phénotype du chondrocyte.

Pour éviter toute adhérence des chondrocytes à un substrat, diverses techniques de culture en suspension ont été mises au point. Les cellules peuvent être enrobées dans une solution de méthylcellulose [24]. Dans des bouteilles en agitation, les chondrocytes forment des agrégats et ce modèle a été utilisé pour des études pharmacologiques sur des chondrocytes humains [25]. On peut aussi apporter une matrice tridimensionnelle exogène aux chondrocytes en incluant ceux-ci dans un gel de collagène [26] ou d'agar [21], mais la plupart des études ont été effectuées avec du collagène I qui n'est pas un constituant naturel du cartilage, et qui peut avoir une influence sur l'activité synthétique des chondrocytes. D'autre part, si des chondrocytes dédifférenciés sont propagés en inclusion dans un gel d'agarose [27] ou en suspension sur un gel d'agarose [28], ils cessent leur synthèse de collagène I et recommencent à synthétiser des protéines marqueurs du cartilage, comme les collagènes II et X. Cette plasticité du phénotype chondrocyte a été exploitée chez l'homme dans le cas d'une chondrodysplasie létale où des chondrocytes épiphysaires obtenus à partir d'une biopsie ont d'abord été amplifiés en monocouche, puis redifférenciés en suspension sur agarose. Une mutation dans le gène du collagène II a ensuite été révélée par séquençage de l'ADNc synthétisé à partir de l'ARN de ces cellules [29]. La culture en agarose a aussi été exploitée pour montrer que

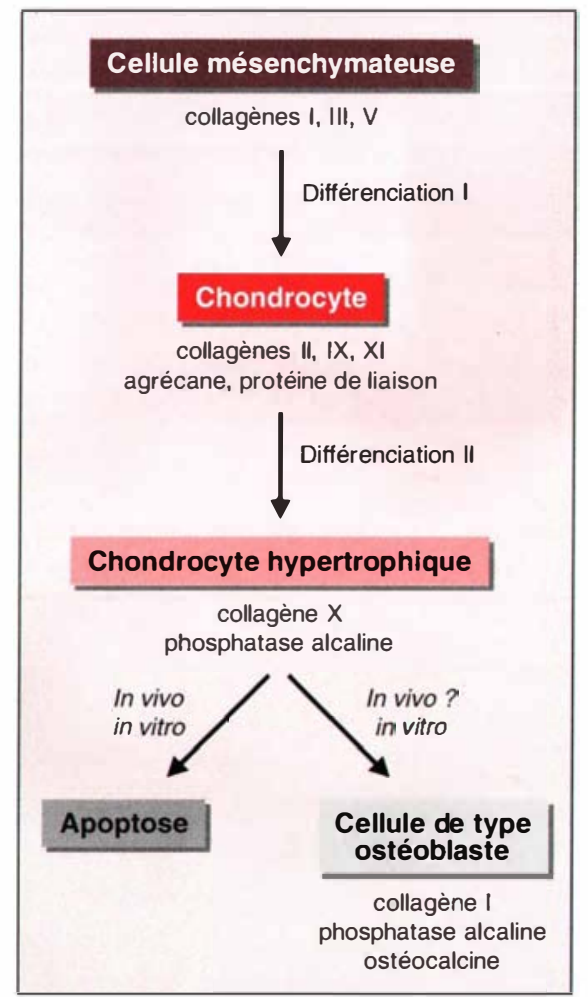

Figure 3. Étapes de la différenciation du chondrocyte. Les différents marqueurs caractérisant chaque phénotype sont mentionnés. La différenciation I aboutit au phénotype des chondrocytes du cartilage articulaire. Au cours de l'ossification endochondrale, le chondrocyte entreprend une différenciation II dans le cartilage de croissance. La différenciation du chondrocyte hypertrophique en cellule de type préostéoblastique n'a été démontrée qu'en culture cellulaire ou organotypique.

des chondrocytes issus d'un foetus atteint d'achondrogenèse de type IB synthétisent du collagène I, qui n'est pas normalement présent dans le cartilage [30]. Récemment, un nouveau système tridimensionnel de culture de chondrocytes a été développé dans lequel les cellules sont encapsulées dans l'alginate, un copolymère d'acide D-mannuronique et L-guluronique. Dans ces conditions, les cellules maintiennent leur phénotype et élaborent une matrice de type cartilage [31]. L'avantage de ce système est que l'on peut récupérer les chondrocytes en solubilisant l'alginate avec des agents chélatants et isoler les ARN, alors que l'extraction d'ARN à partir de cellules incluses en agarose s'est toujours avé- 


\section{RÉFÉRENCES}

40. Poliard A, Lamblin D, Marie PJ, BucCaron $\mathrm{MH}$, Kellermann $\mathrm{O}$. Commitment of the teratocarcinoma-derived mesodermal clone Cl towards terminal osteogenic differentiation. J Cell Sci 1993; 106: 503-12.

41. Poliard A, Nifuji A, Lamblin D, Plee E, Forest C, Kellermann O. Controlled conversion of an immortalized mesodermal progenitor cell towards osteogenic, chondrogenic, or adipogenic pathways. J Cell Biol 1995 ; 130: 1461-72.

42. Enomoto MI, Takigawa M. Regulation of tumor-derived and immortalized chondrocytes. In: Adolphe M, ed. Biological regulation of chondrocyte. New York: CRC Press, 1992: 321-38.

43. Zhao GQ, Zhou X, Eberspaecher H, Solursh $M$, de Crombrugghe $B$. Cartilage homeoprotein 1, a homeoprotein selectively expressed in chondrocytes. Proc Nat Acad Sci USA 1993; 90: 8633-8637.

44. Takigawa M, Pan HO, Kinoshita A Tajima K Takano Y. Establishment from human chondrosarcoma of a new immortal cell line with high tumorigenicity in vivo, which is able to form proteoglycan-rich cartilage-like nodules and to respond to insulin in vitro. Int J Cancer 1991 ; 48: 717-27.

45. Horton WE, Jr, Cleveland J, Rapp U, Nemuth G, Bolander M, Doege K, Yamada Y, Hassell JR. An established rat cell line expressing chondrocyte properties. Exp Cell Res $1988 ; 178$ : 457-68.

46. Oxford JT, Doege KJ, Horton WE, Jr, Morris NP. Characterization of type II and type XI collagen synthesis by an immortalized rat chondrocyte cell line (IRC) having a low level of type II collagen mRNA expression. Exp Cell Res 1994; 213: 28-36.

47. Mallein-Gerin F, Olsen BR. Expression of simian virus 40 large $T$ (tumor) oncogene in mouse chondrocytes induces cell proliferation without loss of the differentiated phenotype. Proc Natl Acad Sci USA 1993 $90: 3289-93$.

48. Mallein-Gerin F, Ruggiero F, Quinn TM Bard F, Grodzinsky AJ, Olsen BR, Van der Rest M. Analysis of collagen synthesis and assembly in culture by immortalized mouse chondrocytes in the presence or absence of al(IX) collagen chains. Exp Cell Res 1995; 218: 257-65.

49. Fässler R, Schnegelsberg PNJ, Dausman J, Shinya T, Muragaki Y, McCarthy MT, Olsen BR, Jaenisch R. Mice lacking $\alpha 1$ (IX) collagen develop noninflammatory degenerative joint disease. Proc Natl Acad Sci USA 1994 ; $91: 5070-4$

50. Nakata K, Ono K, Miyazaki JL, Olsen BR, Muragaki Y, Adachi E, Yamamura KI, Kimura T. Osteoarthritis associated with mild chondrodysplasia in transgenic mice expressing $\alpha 1(\mathrm{IX})$ collagen chains with a central deletion. Proc Natl Acad Sci USA $1993 ; 90: 2870-4$.

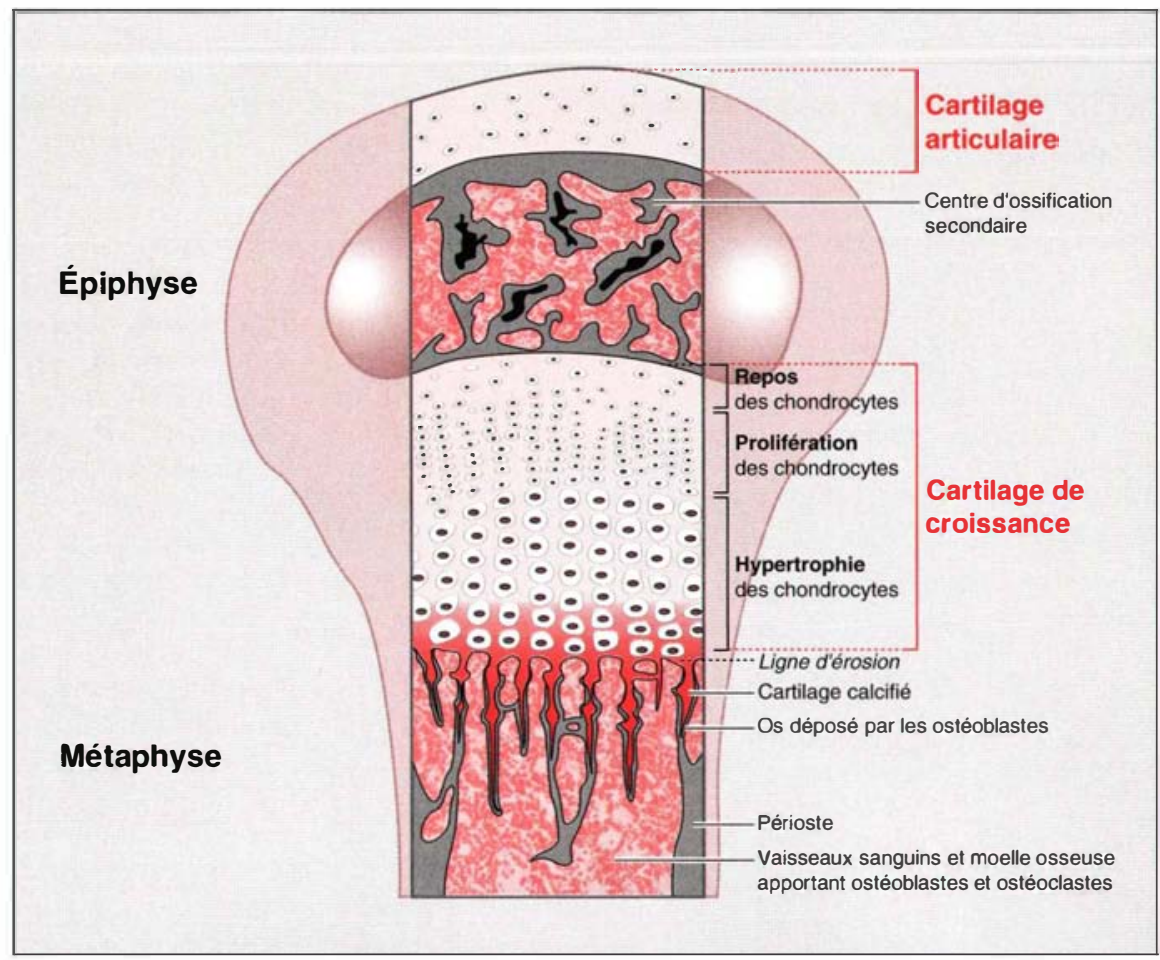

Figure 4. Représentation schématique du cartilage épyphisaire en développement. Les chondrocytes du cartilage de croissance sont organisés en colonnes, avec une matrice extracellulaire qui se calcifie près de la ligne $d^{\prime}$ érosion. A ce niveau, les chondrocytes hypertrophiques meurent par apoptose, laissant des travées de cartilage calcifié sur lesquelles les ostéoblastes déposent une matrice osseuse. C'est le processus d'ossification endochondrale, qui se produit aussi de façon radiaire dans l'épiphyse, et qui aboutit à la formation des os courts et longs.

rée difficile. Des chondrocytes humains, préalablement dédifférenciés en monocouche, réexpriment dans l'alginate les gènes codant pour le collagène II ou l'agrécane [32] et ce système pourrait aussi être appliqué dans des études pathologiques.

Finalement, la culture organotypique de tranches de cartilage permet l'étude des chondrocytes dans leur environnement naturel, puisque le tissu demeure intact. Cette technique a permis, par exemple, de comparer le métabolisme de l'agrécane après marquage métabolique dans le cartilage humain normal et arthrosique [33].

\section{Contrôle par les facteurs solubles dans l'environnement}

Le rôle des facteurs de croissance a été largement étudié au cours de la différenciation du cartilage in vivo et in vitro [34]. Les études in vivo sont cependant difficiles à interpréter car des composés systémiques peuvent exercer des effets concurrents. De même, les études in vitro sont généralement conduites en présence de sérum, dont les composants peuvent interférer avec le facteur ajouté et étudié. Pour éviter cette interférence, des conditions de culture sans sérum ont été développées [35]. D’une façon générale, l'IGF (insulin-like growth factor) et le TGF 3 (transforming growth factor $\beta$ ) sont de puissants stimulants de la synthèse de l'ADN et augmentent la synthèse des protéoglycanes et des collagènes. Le bFGF (basic fibroblastic growth factor) et le PDGF (platelet derived growth factor) ont une action essentiellement mitogène. La thyroxine stimule l'hypertrophie et la synthèse de collagène de type X. Récemment, on s'est intéressé à une famille de protéines morphogènes du cartilage (CDMP: cartilage derived morphogenetic proteins) et 


\section{RÉFÉRENCES}

51. Alema S, Tato F, Boettiger D. $M y c$ and $s r c$ oncogenes have complementary effects on cell proliferation and expression of specific extracellular matrix components in definitive chondroblasts. Mol Cell Biol 1985 . $5: 538-44$.

52. Gionti E, Pontarelli G, Cancedda R. Avian myelocytomatosis virus immortalizes differentiated quail chondrocytes. Proc Natl Acad Sci USA 1985; 82 : 2756-60.

53. Quarto R, Dozin B, Tacchetti C, Robino G, Zenke M, Campanile G, Cancedda R. Constitutive myc expression impairs hypertrophy and calcification in cartilage. Dev Biol 1992; 149 : 168-76.

54. Iwamoto $M$, Yagami $K$, Lu Valle $P$, Olsen BR, Petropoulos CI, Ewert DL, Pacifici M. Expression and role of c-myc in chondrocytes undergoing endochondral ossification. J Biol Chem 1993; 268: 9645-52.

55. Farquharson C, Hesketh JE, Loveridge $\mathrm{N}$. The proto-oncogene $c-m y c$ is involved in cell differentiation as well as cell proliferation: studies on growth plate chondrocytes in situ. J Cell Physiol 1992; 152 : 135-44.

56. Thenet S, Benya PD, Demignot S, Feunteun J, Adolphe M. SV40-immortalization of rabbit articular chondrocytes: alteration of differentiated functions. [ Cell Physiol 1992; $150: 158-67$.

57. Goldring MB, Birkhead JR, Suen LF, Yamin R, Mizuno S, Glowacki J, Arbiser JL, Apperley JF. Interleukin-1 $\beta$-modulated gene expression in immortalized human chondrocytes. J Clin Invest 1994; 2307-16.

58. Lefebvre V, Garofalo S, de Crombrugghe $B$. Type $X$ collagen gene expression in mouse chondrocytes immortalized by a temperature-sensitive simian virus 40 large tumor antigen. I Cell Biol 1995; 128: 239-45.

59. Cheah KSE, Levy A, Trainor PA, Wai AWK, Kuffner T, So CL, Leung KKH, Lovell-Badge RH, Tam PPL. Human COL2A1-directed SV40 T antigen expression in transgenic and chimeric mice results in abnormal skeletal development. J Cell Biol 1995 ; 128: 223-37.

60. Brittberg M, Lindahl A, Nilsson A, Ohlsson $\mathrm{C}$, Isaksson $\mathrm{O}$, Peterson L. Treatment of deep cartilage defects in the knee with autologous chondrocyte transplantation. NEngl J Med 1994; 331 : 889-95.

61. Pereira RF, Halford KW, O'Hara MD, Leeper DB, Sokolov BP, Pollard MD, Bagasra O, Prockop DJ. Cultured adherent cells from marrow can serve as long-lasting precursor cells for bone, cartilage, and lung in irradiated mice. Proc Natl Acad Sci USA $1995 ; 92$ : 4857-61.

62. Rebel A, Baslé M, Racadot I. Cartilage, os, ossification. In: Coujard R, Poirier J, Racadot J, eds. Précis d'histologie humaine. Paris : Masson, 1980: 205-50.

63. Heinegard D, Oldberg A. Glycosylated matrix proteins. In: Royce PM, Steinmann $\mathrm{B}$, eds. Connective tissue and its heritable disorders. New York: Wiley-Liss Inc, 1993: 189 209.

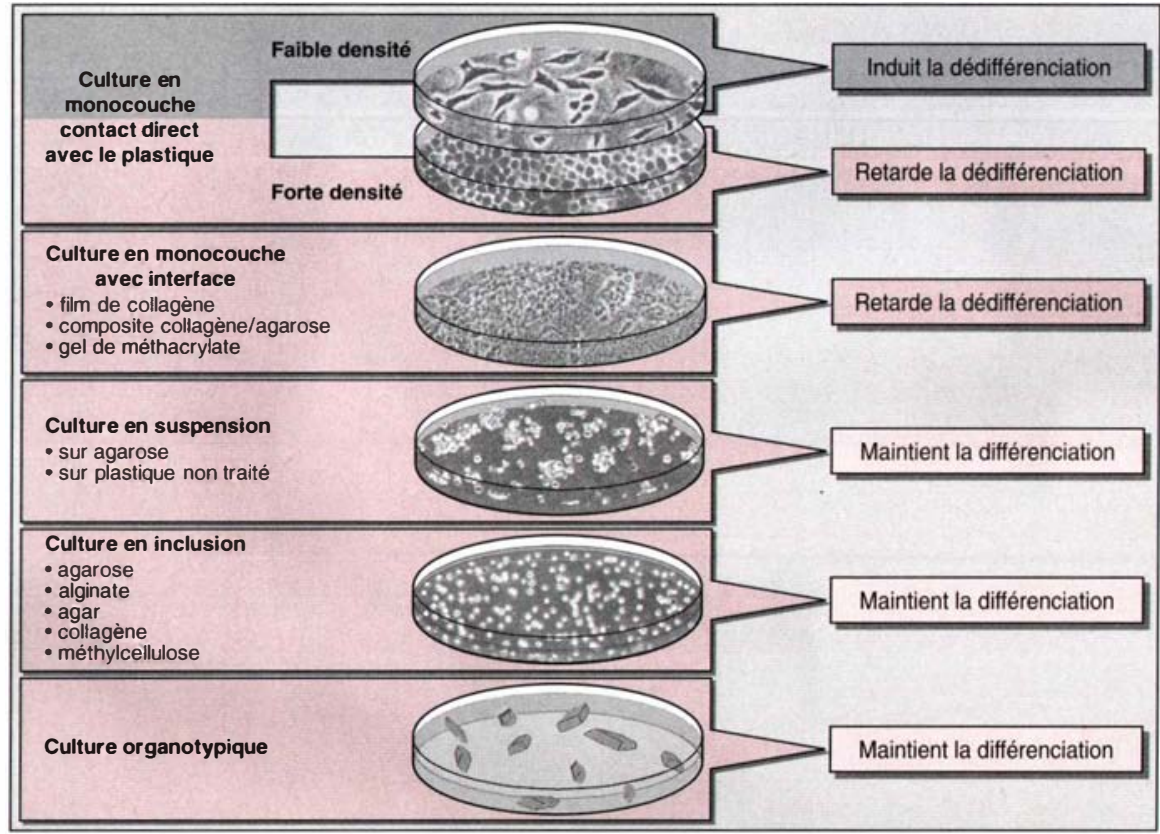

Figure 5. Les différents types de culture de chondrocytes, avec les effets généralement observés sur le phénotype des chondrocytes. II existe cependant une variabilité de ces effets selon le type de collagène ou le type de facteurs solubles ajoutés.

de l'os (BMP: bone morphogenetic proteins), qui jouent un rôle dans le développement du squelette in vive [36]. Les BMP, qui appartiennent à la super-famille du TGF $\beta$, peuvent aussi déclencher la chondrogenèse et empêcher la dédifférenciation in vitro. Ainsi, facteurs de croissance, vitamines, hormones et cytokines peuvent être ajoutés pour orienter le phénotype du chondrocyte in vitr (Tableau II)

\section{Lignées de cellules mésenchymateuses à potentiel chondrogénique}

Les chondrocytes dérivent de cellules mésenchymateuses pluripotentes et des lignées mésenchymateuses précurseurs de cartilage ont été obtenues. Un traitement par la 5-Azacytidine convertit la lignée fibroblastique embryonnaire de souris $(10 \mathrm{Tl} / 2)$ en fibres musculaires, adipocytes et chondrocytes [37]. L'ajout de BMP2 dans cette lignée provoque une différenciation dépendante de la dose en adipocytes, chondrocytes, et ostéoblastes [38]. La transfection permanente des gènes codant pour BMP2 et BMP4 dans les cellules $10 \mathrm{Tl} / 2$ induit la différenciation en ostéoblastes, chondro- cytes, et adipocytes [39]. Le clone C1 dérivé d'un tératocarcinome murin est capable de se différencier en ostéoblastes en présence d'acide ascorbique et de $\beta$-glycérophosphate, si les cellules forment des agrégats en suspension [40]. L'ajout de dexaméthasone convertit les cellules $\mathrm{Cl}$ en chondrocytes et l'action synergique de dexaméthasone et d'insuline convertit les mêmes cellules en adipocytes [41]. Ces systèmes cellulaires inductibles représentent des modèles intéressants pour analyser le lignage des cellules d'origine mésodermique comme les chondrocytes. Cependant, ces lignées produisent une quantité limitée de chondrocytes, quelquefois diluée parmi d'autres phénotypes et après un délai de conversion souvent long.

\section{Lignées établies à partir de chondrosarcomes}

Le chondrosarcome de Swarm est une tumeur apparue spontanément chez un rat et qui a été maintenue par transplantation sous-cutanée. Les cellules de cette tumeur synthétisent du collagène II et l'agrécane du cartilage [42]. A partir de cette tumeur, la technique sensible de PCR (polymerase 
Tableau II

FACTEURS SOLUBLES INTERVENANT DANS LA SQUELETTOGENĖSE IN VIVO, ET MODULANT LE PHÉNOTYPE DU CHONDROCYTE IN VITRO (LISTE NON EXHAUSTIVE)

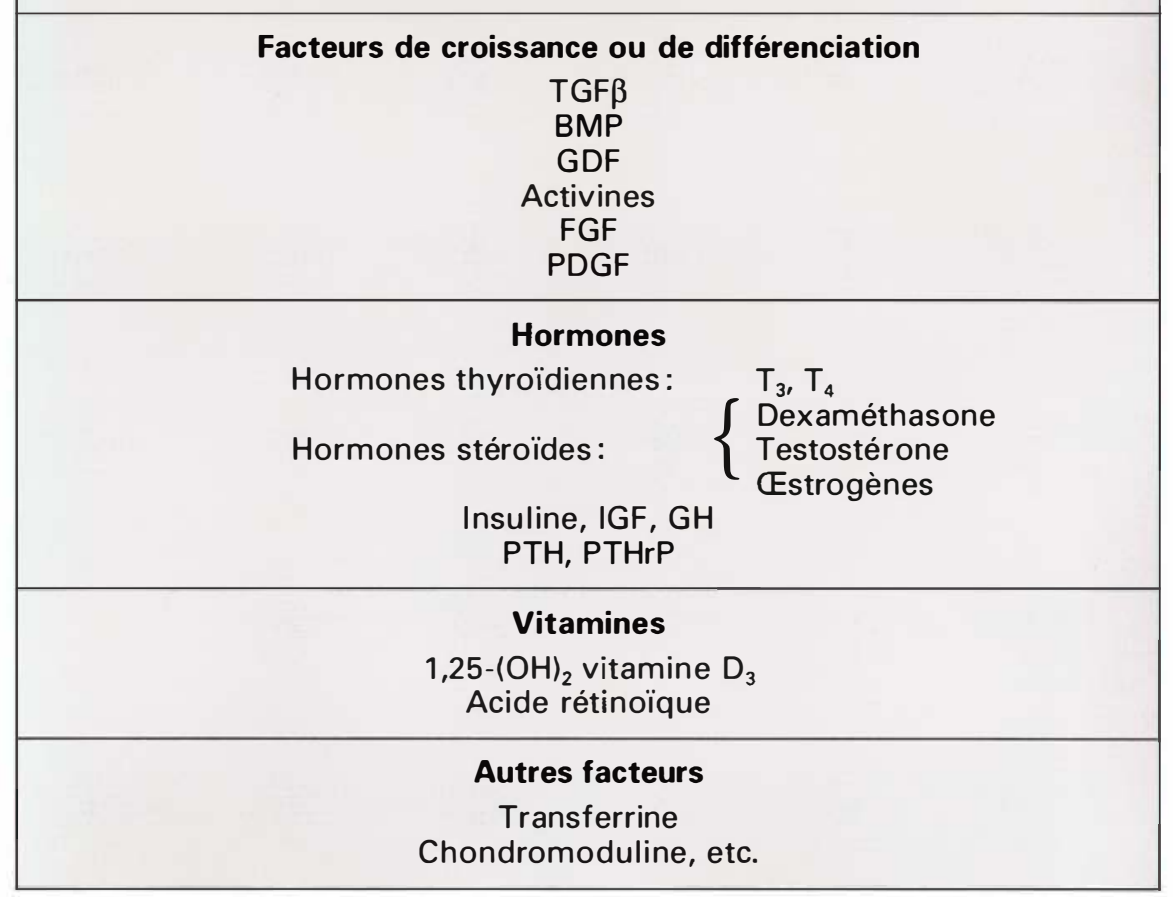

II existe plusieurs isoformes pour la plupart de ces facteurs, qui n'ont pas la même activité suivant l'état de différenciation des cellules. De plus, l'effet d'un facteur peut être différent in vitro selon la présence ou non de sérum. L'acide rétinoïque utilisé à faible ou forte concentration n'a également pas le même effet. TGF $\beta$ : transforming growth factor $\beta, B M P$ : bone morphogenetic protein, GDF: growth differentiation factor, FGF: fibroblast growth factor, PDGF: platelet derived growth factor, PTH(rP): para-thyroid-hormone (related peptide).

chain reaction) a permis d'identifier un ADNc codant pour une protéine homéotique (facteur de transcription), dont l'expression coïncide avec la différenciation du mésenchyme en cartilage [43]. A partir de chondrosarcomes humains, d'autres lignées ont pu être établies avec un phénotype cartilage [44]. D'une façon générale, cependant, les cellules de ces chondrosarcomes se divisent lentement en culture, ce qui rend les expérimentations laborieuses.

\section{Immortalisation} de chondrocytes

Des oncogènes à pouvoir immortalisant ont été utilisés pour obtenir des lignées à partir de chondrocytes en culture. Ces immortalisations conduisent parfois à une perte du phénotype différencié et seuls seront exposés ici les travaux dans lesquels le phénotype a pu être préservé et qui

deux aspects de la biologie du cartilage: la synthèse et l'organisation de macromolécules de structure de la matrice extracellulaire et la relation existant entre le cycle cellulaire et la maturation du chondrocyte.

\section{Synthèse et assemblage \\ des macromolécules du cartilage}

Des chondrocytes embryonnaires de rat ont été immortalisés avec l'oncogène $v$-myc porté par un rétrovirus recombinant. Ces cellules synthétisent l'agrécane, mais les ARNm codant pour la chaîne $\alpha$ l du collagène II représentent seulement $1 \%$ de la quantité synthétisée par des chondrocytes non infectés [45]. Cette propriété a été exploitée pour analyser l'assemblage des chaînes $\alpha$ des collagènes II et XI. Le collagène II est un homotrimère composé de chaînes $\alpha$ l(II) alors que le collagène XI est un hétérotrimère composé des chaînes $\alpha \mathrm{l}(\mathrm{XI})$, $\alpha 2$ (XI) et $\alpha 3(\mathrm{XI})$, les chaînes $\alpha$ l(II) et $\alpha 3(\mathrm{XI})$, codées par le même gène, sont équivalentes. Lorsque les chaînes $\alpha$ l(II) sont présentes en faible quantité, elles s'associent préférentiellement aux chaînes $\alpha \mathrm{l}$ (XI) et $\alpha 2(\mathrm{XI})$ pour former uniquement du collagène XI. Le traitement des cellules immortalisées par de l'arginine augmente le niveau de synthèse des chaînes $\alpha$ l(II) qui deviennent alors majoritaires et s'assemblent en homotrimères pour former du collagène II [46].

Des chondrocytes embryonnaires de souris ont été infectés par un rétrovirus portant l'antigène grand $\mathrm{T}$ de SV40 et les cellules prolifèrent en conservant pendant plusieurs mois l'expression du phénotype cartilage [47]. En suspension, les cellules reconstituent une matrice extracellulaire dont l'observation en microscopie électronique révèle des fibres décorées avec un anticorps anti-collagène II [48], habituellement présentes dans le cartilage in vivo. Cette étude a montré que l'immortalisation de chondrocytes de souris permettait l'analyse de la matrice cartilagineuse in vitro. La même stratégie a été utilisée avec des chondrocytes de souris transgéniques ne produisant plus la chaîne $\alpha$ l du collagène IX après invalidation du gène correspondant [49], afin de mieux comprendre le rôle du collagène IX dans la fibrillogenèse du collagène et/ou dans la structure du cartilage. Le collagène IX est composé de trois chaînes distinctes $\alpha 1, \alpha 2$, et $\alpha 3$ et sa localisation à la surface des fibres de collagène a fait supposer que cette molécule est impliquée dans l'interaction des fibres entre elles ou avec d'autres composants de la matrice. Les chondrocytes déficients en collagène $\alpha \mathrm{l}$ (IX) et immortalisés par l'oncogène T de SV40 synthétisent du collagène II et reconstituent également en suspension une matrice de type cartilage, mais les fibres de collagène semblent fusionner entre elles, suggérant que le collagène IX sert d'espaceur entre les fibres pour éviter un effondrement de la matrice [48]. Ces résultats complètent les études in vivo où les souris ne synthétisant pas la chaîne $\alpha$ l(IX) ou synthétisant la chaîne $\alpha$ l(IX) avec une délétion centrale développent une arthrose avec l'âge, impliquant un rôle de cette chaîne $\alpha 1$ (IX) dans l'intégrité structurale du cartilage $[49,50]$. 


\section{Compatibilité entre division et différenciation du chondrocyte}

Des chondrocytes embryonnaires de poulet et de caille ont pu être immortalisés après infection par un rétrovirus portant l'oncogène myc [51, 52]. Ces cellules synthétisent le collagène II et l'agrécane, mais pas le collagène $\mathrm{X}$. Une autre étude a montré que des chondrocytes de caille non infectés sont capables, en suspension, de s'hypertrophier et de synthétiser du collagène $\mathrm{X}$, et de reconstituer un tissu cartilagineux calcifié [53]. En revanche, si les chondrocytes de caille sont infectés par un virus portant $m y c$, le cartilage reconstitué en suspension par ces cellules ne montre pas de signe d'hypertrophie, ni de calcification. L'absence également de synthèse de collagène $\mathrm{X}$ dans ce dernier cas suggère que c'est l'expression de myc qui entraîne le bloquage de la maturation des chondrocytes [53]. Plus récemment, il a été montré que la surexpression de myc par un rétrovirus dans des chondrocytes de poulet conduit à une répression directe ou indirecte du promoteur du collagène $\mathrm{X}$ [54]. Dans le sternum d'embryon de poulet, l'expression endogène de $c$ myc coïncide topographiquement avec les zones où les cellules prolifèrent et où le collagène $\mathrm{X}$ n'est pas synthétisé. Dans la région céphalique du sternum où les chondrocytes s'hypertrophient, la prolifération cellulaire et l'expression de $c-m y c$ sont à peine détectées, ce qui suggère effectivement une implication de $c-m y c$ dans la régulation physiologique normale du chondrocyte [54]. Chez le poulet et le rat, la protéine c-Myc est détectée par immunohistochimie dans le cartilage de croissance, sauf dans la zone calcifiée, où les chondrocytes hypertrophiques sont en phase terminale de maturation [55]. L'immortalisation de chondrocytes articulaires de lapin avec l'antigène T de SV40 conduit à une altération du phénotype [56] mais il a été montré également que l'expression de cet oncogène dans des chondrocytes murins et humains induit la prolifération cellulaire sans la perte d'expression des collagènes II, IX, XI et de l'agrécane [48, 57]. En revanche, aucun signe d'hypertrophie ni de synthèse de collagène $\mathrm{X}$ n'ont été détec- tés dans ces cellules immortalisées. Par la suite, des chondrocytes murins ont été immortalisés avec un antigène grand T de SV40 thermosensible: les cellules prolifèrent à la température permissive et arrêtent leur croissance à la température restrictive. Dans tous les clones analysés, le passage à la température restrictive s'accompagne d'une augmentation de l'expression des gènes codant pour le collagène $\mathrm{X}$, l'ostéopontine et l'ostéocalcine, marqueurs des chondrocytes hypertrophiques et des ostéoblastes [58]. L'expression ciblée de l'oncogène $\mathrm{T}$ de SV40 sous le contrôle du promoteur du collagène II entraîne chez la souris un développement squelettique anormal avec une absence de synthèse de collagène $\mathrm{X}$ dans le cartilage de croissance [59]. Il apparaît ainsi que l'absence significative de collagène $\mathrm{X}$ dans les lignées de chondrocytes immortalisés par les oncogènes $m y c$ ou grand T de SV40 est due à un niveau de prolifération élevé dans ces cellules. L'utilisation d'oncogènes a donc mis en évidence que l'expression du phénotype cartilage n'est pas incompatible avec la prolifération cellulaire, mais que le chondrocyte doit quitter son activité mitotique pour entreprendre son hypertrophie et exprimer pleinement du collagène $\mathrm{X}$.

\section{Perspectives}

Le résumé des travaux exposé ci-dessus montre que le phénotype du chondrocyte peut être contrôlé en culture. Ce contrôle a permis de créer des outils d'étude in vitro qui ont déjà contribué à mieux faire connaître les mécanismes de différenciation et l'organisation structurale du cartilage. Les avancées récentes de la biologie moléculaire et cellulaire combinées à l'utilisation d'oncogènes ouvrent des perspectives dans le domaine de la recherche fondamentale et appliquée. Ainsi, des lignées stables ou inductibles de chondrocytes représentent des systèmes très utiles pour étudier la régulation des gènes codant pour des protéines caractéristiques du cartilage. Ces systèmes pourraient être également utilisés pour identifier des gènes exprimés de façon différentielle et en faible quantité entre deux états de différenciation, par des stratégies d'hybridation soustractive ou de PCR comparative. L'immortalisation de chondrocytes humains fournit également un modèle cellulaire illimité qui permet de tester divers facteurs pharmacologiques, sous différentes conditions de culture mimant des conditions pathologiques observées in vivo. L'immortalisation de chondrocytes humains de cartilage pathologique (arthrosique par exemple) est aussi envisageable et permettrait de comparer l'expression génique des cellules normales et pathologiques. Ainsi, la combinaison de stratégies d'immortalisation et de conditions de culture bien contrôlées devrait permettre d'identifier plus facilement des facteurs impliqués dans l'établissement ou le dérèglement du phénotype cartilage. Les effets de mutations géniques sur l'organisation moléculaire du cartilage peuvent être aussi analysés en culture tridimensionnelle. Récemment, des chondrocytes humains autologues préalablement amplifiés in vitro ont été transplantés sur des lésions arthrosiques du genou et une reconstitution de cartilage a été observée dans certains cas [60]. Des cellules souches à potentiel chondrogénique dérivées de la moelle osseuse de diverses espèces animales peuvent être amplifiées in vitro et parfois réimplantées [61]. Ces cellules représenteraient également pour l'homme un réservoir important pour une thérapie cellulaire du cartilage. Enfin, la technologie des biomatériaux combinée à celle du transfert de gènes dans les cellules devraient faire progresser rapidement les stratégies thérapeutiques. Des matrices biologiques contenant des cellules aptes à exprimer des facteurs réparateurs pourraient être préparées ex vivo avant leur greffe sur un cartilage déficient

\section{Remerciements}

Les auteurs tiennent à remercier Daniel Herbage pour une lecture critique du manuscrit et Christian Van Herrewege pour l'élaboration des figures. Les travaux du laboratoire concernant l'immortalisation des chondrocytes bénéficient du soutien financier de l'ARC (Association pour la Recherche sur le Cancer).

\section{TIRÉS À PART}

F. Mallein-Gerin. 


\section{Summary}

Analysis of the chondrocyte phenotype in culture

Cartilage differentiation is a multistep process starting from mesenchymal cells and eventually resulting in mature hypertrophic chondrocytes, a stage reached only in cartilage undergoing endochondral ossification. This differentiation is characterized by a switch of gene activation. Mesenchymal cells produce collagens I and III, whereas chondrocytes synthesize the large proteoglycan agrecan and collagens II, IX, and XI. Hypertrophic chondrocytes produce collagen X, a biochemical marker for this stage of cell maturation. This critical developmental pathway of the skeleton is under environmental control of growth factors, hormones, cytokines, extracellular matrix, which have been extensively studied these past years. However, the understanding of the molecular mechanisms of chondrocyte gene regulation or of the precise function of cartilage matrix components has been hindered because of the instability of primary chondrocytes in traditional cul- ture. Suspension culture methods and immortalization strategies have been developed and have allowed a better insight into the mechanisms of synthesis and assembly of cartilage macromolecules. The use of immortalizing oncogenes have also shown that growth and maturation are mutually exclusive phenomena for chondrocytes. These chondrocyte cell lines could be used to clone genes controling the pathway of cartilage differentiation. Furthermore, immortalization of normal human chondrocytes represent a valuable tool to test pharmacological factors, and the potential exists for immortalizing chondrocytes from diseased cartilage. This approach should be useful to identify genes involved in maintenance or disruption of the cartilage phenotype. Taken together, in vitro chondrocyte studies supported by the recent developments of cell and molecular biology should help to better understand the mechanisms of skeletogenesis.

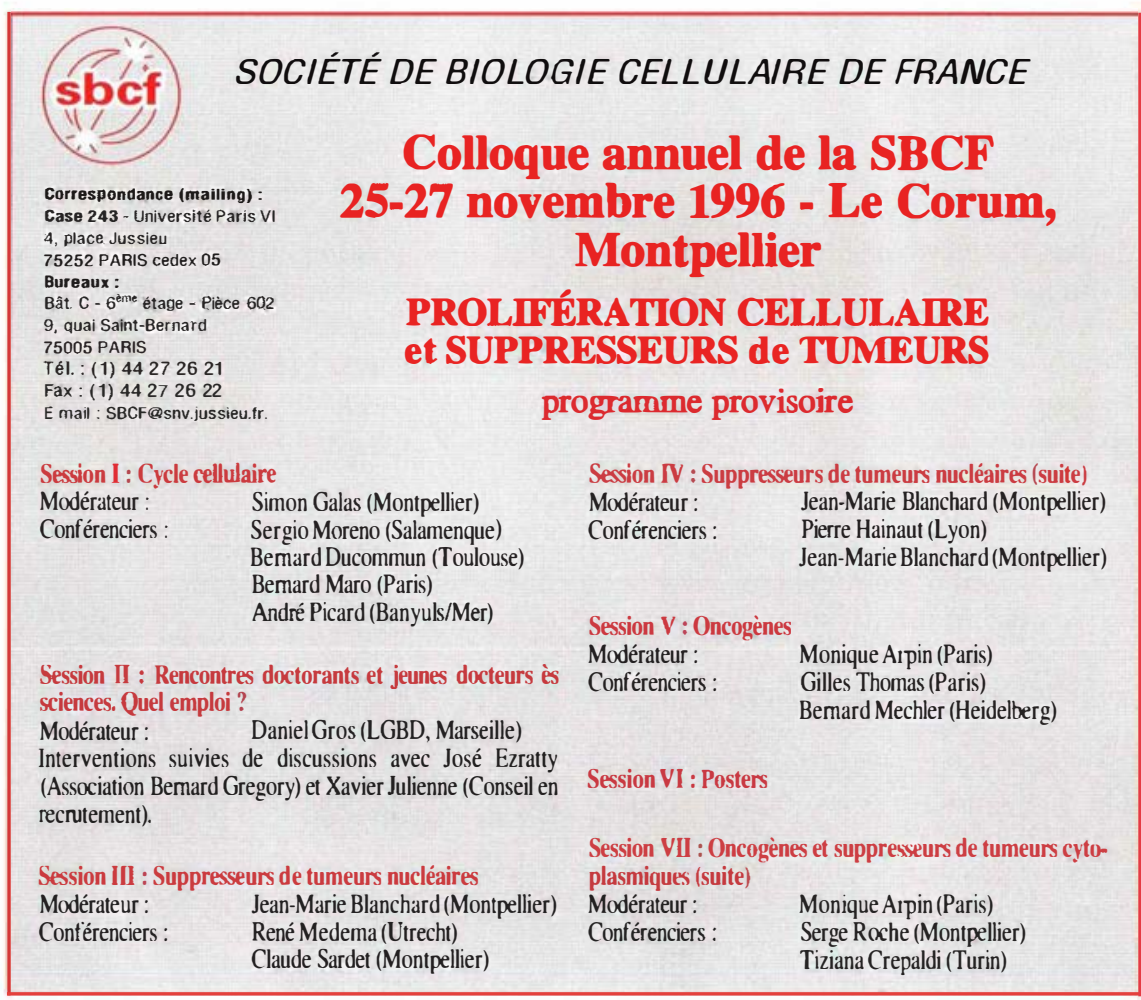

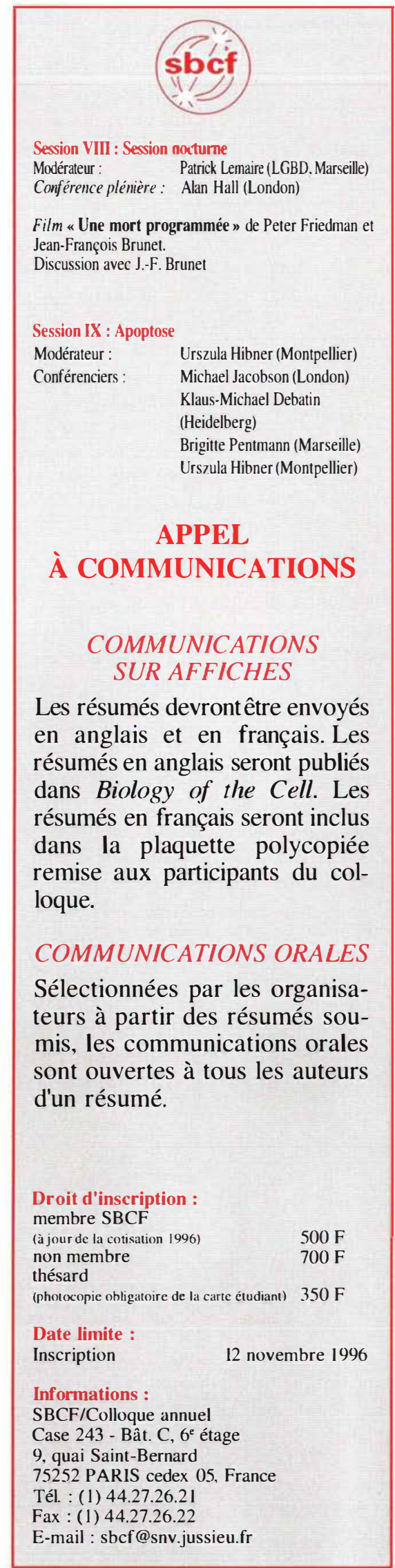

